\title{
EFEITO RESIDUAL DA ADUBAÇÃO NA CANA-PLANTA E DA ADUBAÇÃO NITROGENADA E POTÁSSICA NA CANA-SOCA COLHIDAS COM E SEM A QUEIMA DA PALHADA ${ }^{(1)}$
}

\author{
Nivaldo Schultz ${ }^{(2)}$, Eduardo Lima ${ }^{(3)}$, Marcos Gervasio Pereira $^{(4)} \&$ \\ Everaldo Zonta ${ }^{(5)}$
}

\begin{abstract}
RESUMO
A aplicação de vinhaça na cana-de-açúcar, complementada com adubação nitrogenada e manutenção da palhada na superfície do solo, melhoram as propriedades físicas, químicas e biológicas do solo, podendo resultar em aumento da produtividade da cultura. $O$ objetivo deste estudo foi avaliar o efeito residual de adubação na cana-planta e a adubação da cana-soca, com e sem aplicação de vinhaça, sob diferentes sistemas de colheita no município de Conceição da Barra ES. O experimento foi conduzido no período de 2005 a 2007, num Argissolo Amarelo em Tabuleiros Costeiros. Utilizou-se o delineamento de blocos ao acaso com quatro repetições, no esquema fatorial $2 \times 7$, com parcelas subdivididas, sendo dois tratamentos cana crua e cana queimada e sete subtratamentos com diferentes doses de $\mathrm{N}$ associadas a doses de $\mathrm{K}_{2} \mathrm{O}$ na forma de cloreto de $\mathrm{K}$ e vinhaça. Os tratamentos foram: testemunha (T1); vinhaça (T2); vinhaça $+80 \mathrm{~kg}$ de $\mathrm{N}$ incorporado (T3); vinhaça $+80 \mathrm{~kg}$ de $\mathrm{N}$ em cobertura (T4); vinhaça $+40 \mathrm{~kg}$ de $\mathrm{N} \mathrm{em}$ cobertura (T5); $\mathrm{K}_{2} \mathrm{O}+80 \mathrm{~kg}$ de $\mathrm{N}$ incorporado (T6); e $\mathrm{K}_{2} \mathrm{O}+80 \mathrm{~kg}$ de $\mathrm{N}$ em cobertura (T7). Não houve efeito residual de adubação na produtividade de colmos na canaplanta. A cana crua apresentou produtividade superior à da cana queimada na cana-soca. Nesta, a fertilização com vinhaça complementada com $\mathrm{N}$ incorporado ou em cobertura e a adubação com $120 \mathrm{~kg}$ de $\mathrm{K}_{2} \mathrm{O}$ na forma de $\mathrm{KCl}+80 \mathrm{~kg}$ de $\mathrm{N}$ incorporados ao solo proporcionaram produtividade superior à dos demais
\end{abstract}

\footnotetext{
(1) Parte da Dissertação de Mestrado do primeiro autor apresentada ao Curso de Pós-Graduação em Agronomia - Ciência do Solo (CPGA-CS) da Universidade Federal Rural do Rio de Janeiro - UFRRJ. Recebido para publicação em maio de 2009 e aprovado em março de 2010.

(2) Mestre em Agronomia, Ciência do Solo, Universidade Federal Rural do Rio de Janeiro - UFRRJ. BR 465 km 7, Departamento de Solos, CEP 23890-000 Seropédica (RJ). E-mail: nsufrrj.br@yahoo.com.br

(3) Professor Adjunto III do Departamento de Solos da UFRRJ. E-mail: edulima@ufrrj.br

(4) Professor Associado II do Departamento de Solos da UFRRJ. Bolsista do CNPq. E-mail:gervasio@ufrrj.br

(5) Professor Adjunto III do Departamento de Solos da UFRRJ. E-mail: edulima@ufrrj.br
} 
tratamentos. Não foi verificada diferença na produtividade de colmos entre as doses de 40 e $80 \mathrm{~kg} \mathrm{ha}^{-1}$ de $\mathrm{N}$, aplicadas em cobertura na cana-soca. A extração e retorno de nutrientes ocorreu de forma similar entre cana-planta e cana-soca.

Termos de indexação: Saccharum sp., vinhaça, cana queimada, cana crua.

\author{
SUMMARY: RESIDUAL EFFECTS OF NITROGEN, POTASSIUM AND \\ VINASSE, FERTILIZATION ON CANE PLANT AND RATOON \\ HARVESTED WITH AND WITHOUT STRAW BURNING
}

\begin{abstract}
The application of vinasse supplemented with nitrogen fertilizer to sugarcane with stubble maintained on the soil surface improves the physical, chemical and biological soil properties and may result in increased crop yield. The aim of this study was to evaluate residual effects of fertilization on cane plant, as wellas the effect of vinasse application to ratoon cane under different management systems in Conceição da Barra, state of Espírito Santo. The experiment was carried out from 2005 to 2007, on an Ultisol on the Coastal Plains, in a randomized block, $2 \times 7$ factorial design with four replications with split plots. The two treatments consisted of green cane and burnt cane and the seven subtreatments of different $\mathrm{N}$ and $\mathrm{K}_{2} \mathrm{O}$ doses as potassium chloride and vinasse, as follows: control (T1), vinasse (T2), vinasse $+80 \mathrm{~kg}$ of incorporated $N$ (T3), vinasse $+80 \mathrm{~kg}$ of $N(T 4)$, vinasse $+40 \mathrm{~kg}$ of sidedressed $\mathrm{N}(\mathrm{T5}), \mathrm{K}_{2} \mathrm{O}+$ $80 \mathrm{~kg}$ of incorporated $\mathrm{N}$ (T6), $\mathrm{K}_{2} \mathrm{O}+80 \mathrm{~kg}$ of sidedressed $\mathrm{N}$ (T7). No residual effect of fertilization on sugarcane yield was observed. The yield of green cane was higher than that of the burnt ratoon cane. For ratoon cane, fertilization with vinasse supplemented with incorporated or sidedressed $\mathrm{N}$ and fertilization with $120 \mathrm{~kg} \mathrm{~K}_{2} \mathrm{O}$ in the form of $\mathrm{KCl}+$ incorporated $80 \mathrm{~kg} N$ achieved best results. No difference in the stalk yield was observed between doses of 40 and $80 \mathrm{~kg} \mathrm{ha}^{-1}$ sidedressed N applied to ratoon cane. Nutrient extraction and return were similar for cane plant and ratoon cane.
\end{abstract}

Index terms: Saccharum sp., vinasse, burnt cane, green cane.

\section{INTRODUÇÃO}

A colheita mecanizada da cana-de-açúcar deixa sobre o solo uma cobertura de resíduos culturais na ordem 10 a 20 t ha ${ }^{-1}$ de material seco, constituída por ponteiros, folhas secas e pedaços de colmo, que promovem a ciclagem de alguns nutrientes, com destaque para o $\mathrm{N}$, com valores variando entre $40 \mathrm{e}$ $60 \mathrm{~kg} \mathrm{ha}^{-1}$, S, de 15 a $30 \mathrm{~kg} \mathrm{ha}^{-1}$, e C, $4.500 \mathrm{~kg} \mathrm{ha}^{-1}$, que irão provocar mudanças significativas no manejo da cultura e influenciar a adubação de soqueiras (Urquiaga et al., 1997; Resende et al., 2006). A manutenção dessa palhada sobre o solo proporciona melhoria em suas propriedades físicas, químicas e biológicas, além de favorecer a sua conservação (Canellas et al., 2003).

A produtividade da cana-de-açúcar está relacionada diretamente à adubação nitrogenada, uma vez que o $\mathrm{N}$ é um dos nutrientes limitantes à produtividade $\mathrm{e}$ longevidade das soqueiras de cana-de-açúcar (Vitti, 2003). Atualmente a adubação nitrogenada não vem sendo recomendada no plantio da cana-de-açúcar, ou, quando recomendada, ela é feita em pequenas doses (30 kg ha-1). No entanto, nas soqueiras, a aplicação se faz necessária, apresentando ampla variação de acordo com o manejo e tipo de solo (Boddey et al., 2001; Urquiaga et al., 2003). Segato et al. (2006) atribuem a ausência de resposta da cana-planta à adubação nitrogenada à mineralização da matéria orgânica que ocorre durante o preparo do solo, às reservas existentes no tolete e ao sistema radicular da cana-de-açúcar, o qual, por ser extenso e profundo, favorece a absorção do nutriente. Além disso, a cana-de-açúcar forma associações com bactérias fixadoras de $\mathrm{N}_{2}$ do ar atmosférico (Döbereiner et al., 1972; Lima et al., 1987; Urquiaga et al., 1992).

A vinhaça constitui o principal efluente das destilarias de álcool, sendo produzidos em média $13 \mathrm{~L}$ de vinhaça para cada litro de álcool. Até no início da década de 1980, toda a vinhaça produzida era depositada nos rios, córregos e outros reservatórios de água (Gloria \& Orlando-Filho, 1984). Hoje em dia, toda a vinhaça produzida na industrialização da canade-açúcar é utilizada como fertilizante, fonte de 
nutrientes, matéria orgânica e água no sistema produtivo da cana-de-açúcar. Avaliando as propriedades químicas de diferentes solos submetidos à aplicação de vinhaça por um período de 20 anos, Orlando Filho et al. (1983) concluíram que não houve efeitos negativos nas propriedades químicas dos solos estudados, verificando-se efeito favorável à aplicação do resíduo, traduzido por elevação dos teores de $\mathrm{K}$, $\mathrm{Ca}, \mathrm{Mg}$, soma de bases, aumento da capacidade de troca de cátions (CTC), elevação do pH, aumento da disponibilidade de alguns íons, aumento da capacidade de retenção de água e melhoria da estrutura do solo.

Relacionando o efeito das aplicações de vinhaça nas doses equivalentes a 0, 100, 200, 400 e $800 \mathrm{~m}^{3} \mathrm{ha}^{-1}$ no pH e Eh do solo, Leal et al. (1983) verificaram que a elevação do $\mathrm{pH}$ foi resultante das reações de redução, nas quais há consumo de $\mathrm{H}^{+}$, que se encontra em condições de predominância no solo. Segundo Amaral Sobrinho (1983), a aplicação de vinhaça complementada com adubação nitrogenada pode causar perdas de $\mathrm{N}$ pelas reações de redução do $\mathrm{NO}_{3}{ }^{-}$a $\mathrm{N}_{2}$. Para De-Polli et al. (1988), o uso da vinhaça como fertilizante deve ser feito de forma criteriosa, pois sua composição química é muito variável, principalmente em função da natureza da matéria-prima originária e do processo industrial de destilação, ou seja, se proveniente do mosto do caldo, do melaço ou resultante da mistura de caldo e melaço, denominada de vinhaça mista. Segundo Rodrigues et al. (1984), a aplicação de doses de vinhaça superiores a $120 \mathrm{~m}^{3} \mathrm{ha}^{-1}$ ao longo de vários anos seguidos pode promover alterações na fisiologia da cana-de-açúcar, como: aumento do teor de umidade dos colmos, redução dos teores de lignina, aumento do fator acamamento, aumento dos teores de K em todas as partes da cana, aumento dos teores de cinzas e redução dos teores de sacarose (Freire \& Cortez, 2000).

O objetivo deste estudo foi avaliar o efeito residual de adubação na cana-planta e adubação nitrogenada da cana-soca, associada à aplicação de vinhaça e $\mathrm{K}_{2} \mathrm{O}$ na forma de cloreto de potássio, sob diferentes sistemas de colheita, no município de Conceição da Barra-ES.

\section{MATERIAL E MÉTODOS}

O estudo foi conduzido num Argissolo Amarelo, na área da destilaria ALCON, localizada no município de Conceição da Barra-ES, nas coordenadas geográficas de $18^{\circ} 53$ ' 56 " $\mathrm{S}$ de latitude, $39^{\circ} 43^{\prime} 56^{\prime}$ "W de longitude, com aproximadamente $7 \mathrm{~m}$ de altitude (IBGE, 2006). A área experimental esteve sob plantio comercial de cana-de-açúcar desde os anos 1980 até 1998. Neste ano, foi isolada para a condução de experimentos com cana-de-açúcar, sendo o primeiro ciclo de experimentação de 1998 a 2005 . O preparo inicial do solo (1998) foi realizado de acordo com a análise de solo e as necessidades da cultura.
Os tratamentos foram implantados após o corte da cana-planta, em 1999. Os tratamentos foram: T1 = testemunha; $\mathrm{T} 2=150 \mathrm{~m}^{3} \mathrm{ha}^{-1}$ de vinhaça; $\mathrm{T} 3=$ $150 \mathrm{~m}^{3} \mathrm{ha}^{-1}$ de vinhaça $+80 \mathrm{~kg} \mathrm{ha}^{-1}$ de N incorporado; $\mathrm{T} 4=150 \mathrm{~m}^{3} \mathrm{ha}^{-1}$ de vinhaça $+80 \mathrm{~kg} \mathrm{ha}^{-1}$ de $\mathrm{N}$ em cobertura; T5 = $150 \mathrm{~m}^{3} \mathrm{ha}^{-1}$ de vinhaça $+40 \mathrm{~kg} \mathrm{ha}^{-1}$ de $\mathrm{N}$ em cobertura; $\mathrm{T} 6=120 \mathrm{~kg}$ de $\mathrm{K}_{2} \mathrm{O}+80 \mathrm{~kg} \mathrm{ha}^{-1}$ de $\mathrm{N}$ incorporado; e T7 $=120 \mathrm{~kg}$ de $\mathrm{K}_{2} \mathrm{O}+80 \mathrm{~kg} \mathrm{ha}^{-1}$ de $\mathrm{N}$ em cobertura. Foi utilizada vinhaça de mosto de caldo. Segundo Prada et al. (1998), a vinhaça de mosto de caldo pode conter em sua composição de 150 a $700 \mathrm{mg} \mathrm{L}^{-1}$ de $\mathrm{N}$ e de 1.200 a $2.100 \mathrm{mg} \mathrm{L}^{-1}$ de $\mathrm{K}_{2} \mathrm{O}$. Com base nessas informações, a aplicação de $150 \mathrm{~m}^{3} \mathrm{ha}^{-1}$ de vinhaça pode adicionar de 22,5 a $105,0 \mathrm{~kg} \mathrm{ha}^{-1}$ de $\mathrm{N}$ e de 180 a $315 \mathrm{~kg} \mathrm{ha}^{-1}$ de $\mathrm{K}_{2} \mathrm{O}$. A dose de vinhaça foi dividida em duas aplicações, correspondendo a uma lâmina de $7,5 \mathrm{~mm}$. A primeira foi realizada imediatamente após a adubação nitrogenada e a segunda, 15 dias depois, para que não ocorresse fitotoxidez na cultura, devido ao excesso de vinhaça. A aplicação de vinhaça foi feita utilizando-se canhão hidráulico, posicionado no carreador central do experimento, com um giro de $180^{\circ}$. Os tratamentos que não receberam vinhaça foram irrigados com água, utilizando a mesma lâmina estabelecida para a vinhaça. A fonte de $\mathrm{K}_{2} \mathrm{O}$ foi o cloreto de potássio ( $\mathrm{KCl}$ ). A fonte de $\mathrm{N}$ utilizada foi a ureia. Nos tratamentos sem queima, os restos culturais foram amontoados, a ureia aplicada ao lado da linha e, em seguida, a palhada foi redistribuída homogeneamente sobre o solo. Nos tratamentos em que o $\mathrm{N}$ foi incorporado, adicionouse uma camada de solo de aproximadamente $5 \mathrm{~cm}$ sobre o fertilizante. A adubação com $\mathrm{KCl}$ foi feita em cobertura, e a variedade utilizada foi a RB867515. O delineamento experimental utilizado foi em blocos ao acaso com quatro repetições, no esquema fatorial $2 \times 7$, com parcelas subdivididas. Cada bloco foi composto por duas parcelas, cana crua e cana queimada, dentro das quais foram aplicados os tratamentos com as diferentes doses de adubação. Cada tratamento foi composto por quatro linhas de $30 \mathrm{~m}$ de comprimento, espaçadas de 1,20 m, sendo utilizadas para avaliação apenas as duas linhas centrais. Ao longo do ciclo de 1998 a 2004 foram realizados seis cortes e os tratamentos repetidos todos os anos após cada corte.

Em maio de 2005 foi realizada a renovação do canavial, sendo feito o preparo mínimo do solo, que consistiu na abertura de sulcos nas entrelinhas e aplicação de $\mathrm{P}$ no fundo dos sulcos $\left(120 \mathrm{~kg} \mathrm{ha}^{-1} \mathrm{de}\right.$ $\mathrm{P}_{2} \mathrm{O}_{5}$, na forma de superfosfato simples). As mudas foram plantadas nos sulcos, não sendo feita qualquer adubação de cobertura na cana-planta, com o objetivo de avaliar o efeito residual das adubações dos anos anteriores. A colheita da cana-planta foi realizada 15 meses após a implantação, em agosto de 2006.

Cerca de um mês após a colheita da cana-planta, foram aplicados, da mesma forma como no ciclo anterior, os tratamentos descritos anteriormente. A cana-soca foi colhida em agosto de 2007. 
A avaliação do rendimento de colmos, palha e pontas foi realizada em duas áreas de $2,4 \mathrm{~m}^{2}$ dentro de cada tratamento. Em cada área foram coletadas duas amostras do material vegetal. Após a pesagem dos colmos, palha e pontas frescos, foram retiradas as subamostras, para quantificação de matéria seca de cada fração e análises de nutrientes. O material vegetal foi seco em estufa de circulação forçada de ar a $65^{\circ} \mathrm{C}$, até peso constante, e posteriormente moído em moinho tipo Wiley. Em seguida, foram determinados os teores de $\mathrm{N}$ (método Kjeldahl), $\mathrm{P}$ (colorimetricamente) e K (fotometria de chama) de cada fração, segundo Malavolta (1989).

Nas análises estatísticas foi utilizado o software SAEG 5.0 para verificação da normalidade e homogeneidade das variâncias. Quanto à análise de variância, foi utilizado o software SISVAR 4.3, com as médias comparadas pelo teste de Scott-Knott a $5 \%$.

\section{RESULTADOS E DISCUSSÃO}

No quadro 1 são apresentados os valores médios de rendimento de colmos e o acúmulo de palhada da cana-planta colhida crua e queimada sob efeito residual de adubação de anos anteriores. Não foram observadas diferenças significativas no rendimento de colmos entre os sistemas de colheita, cana crua e cana queimada, nem dos efeitos residuais de adubações dos

Quadro 1. Rendimento de colmos frescos e matéria seca de palhada de cana-planta crua e queimada com diferentes doses de adubação

\begin{tabular}{|c|c|c|c|c|}
\hline \multirow{3}{*}{ Adubação } & \multicolumn{4}{|c|}{$\begin{array}{c}\text { Efeito de interação sistema } \\
\text { de colheita/adubação }\end{array}$} \\
\hline & \multicolumn{2}{|r|}{ Colmos } & \multicolumn{2}{|c|}{ Palhada } \\
\hline & Crua & Queimada & Crua & Queimad \\
\hline & \multicolumn{4}{|c|}{$-t h a^{-1}$} \\
\hline $\mathrm{T} 1$ & 142,7 & 138,0 & $15,2 \mathrm{a}$ & 12,6 \\
\hline $\mathrm{T} 2$ & 146,0 & 142,7 & $13,0 \mathrm{~b}$ & 12,5 \\
\hline $\mathrm{T} 3$ & 160,0 & 139,3 & 14,4 & 13,0 \\
\hline $\mathrm{T} 4$ & 138,0 & 150,3 & $12,3 \mathrm{~b}$ & 12,6 \\
\hline $\mathrm{T} 5$ & 167,0 & 158,9 & $15,7 \mathrm{a}$ & 13,9 \\
\hline $\mathrm{T} 6$ & 153,0 & 155,7 & $12,7 \mathrm{~b}$ & 14,2 \\
\hline $\mathrm{T} 7$ & 155,0 & 150,7 & $14,1 \mathrm{a}$ & 12,8 \\
\hline $\mathrm{ESC}$ & 151,6 & 148,0 & 13,9 & 13,1 \\
\hline CV $1(\%)$ & \multicolumn{2}{|r|}{16,9} & \multicolumn{2}{|c|}{18,1} \\
\hline CV $2(\%)$ & \multicolumn{2}{|r|}{9,3} & \multicolumn{2}{|c|}{8,2} \\
\hline
\end{tabular}

Valores médios de quatro repetições para efeito de interação e de 28 repetições para efeito de sistema de colheita. A ausência de letras significa que não houve diferença entre as médias. T1: testemunha; T2: vinhaça; T3: vinhaça $+80 \mathrm{~kg}$ de $\mathrm{N}$ incorporado; T4: vinhaça $+80 \mathrm{~kg}$ de $\mathrm{N}$ em cobertura; T5: vinhaça + $40 \mathrm{~kg}$ de $\mathrm{N}$ em cobertura; T6: $\mathrm{K}_{2} \mathrm{O}+80 \mathrm{~kg}$ de $\mathrm{N}$ incorporado; T7: $\mathrm{K}_{2} \mathrm{O}+80 \mathrm{~kg}$ de $\mathrm{N}$ em cobertura. ESC: efeito de sistema de colheita. CV: coeficiente de variação. Teste de Scott-Knott a $5 \%$. anos anteriores. Esses resultados indicam que os resíduos das adubações dos anos anteriores não influenciaram o desenvolvimento e a produtividade da cana-planta. Vários estudos realizados nas diferentes regiões produtoras do Brasil demonstram que a cana-planta não se apresenta responsiva às adubações nitrogenadas. Segundo Azeredo et al. (1986) e Boddey et al. (2001), a adubação nitrogenada em cana-de-açúcar não é recomendada no plantio, ou, quando esta é realizada, as doses devem ser de aproximadamente $30 \mathrm{~kg} \mathrm{ha}^{-1}$ de N.

O rendimento de colmos foi elevado em todos os tratamentos, inclusive na testemunha absoluta. De acordo com o programa de melhoramento genético da cana-de-açúcar (PMGCA-UFV, 2008), esse elevado rendimento pode estar relacionado às características genéticas da variedade (RB867515), com destaque para tolerância à seca e crescimento rápido com alta produtividade. Avaliando o potencial produtivo dessa mesma variedade sob irrigação complementar em Latossolo Vermelho Escuro distrófico no município de Paracatu, noroeste de Minas Gerais, Oliveira et al. (2003) verificaram rendimento de colmos na canaplanta superior ao obtido neste estudo, confirmando o alto potencial produtivo da variedade.

O acúmulo de palhada apresentou variações entre os tratamentos na cana colhida sem queima. De modo geral, houve acúmulo de 10 a 20 t ha $^{-1}$ de matéria seca. Estudando o acúmulo de restos culturais da cana-de-açúcar após a colheita, Urquiaga et al. (1992) encontraram valores dessa mesma magnitude.

No quadro 2 são apresentados a exportação de N, $\mathrm{P}$ e K nos colmos frescos e o acúmulo na matéria seca de palhada e parte aérea total de cana-planta.

Quanto à exportação de nutrientes nos colmos frescos, não foram observadas diferenças significativas nos valores de $\mathrm{N}$ e $\mathrm{P}$, entre os sistemas de colheita e os resíduos de adubações dos anos anteriores. A exportação de K nos colmos apresentou oscilações no efeito residual de adubação na cana crua, porém as variações observadas parecem ser de fatores externos e não influência dos resíduos de adubações anteriores. Os valores de $\mathrm{N}$ e $\mathrm{P}$ exportados nos colmos foram similares aos obtidos por Oliveira (2008), que, estudando a dinâmica de nutrientes em cana-deaçúcar em sistema irrigado de produção com a mesma variedade verificou a exportação de 152,33 $\mathrm{kg} \mathrm{ha}^{-1} \mathrm{de}$ $\mathrm{Ne} 18,4 \mathrm{~kg} \mathrm{ha}^{-1}$ de P. Os valores de K foram superiores aos observados pelo mesmo autor, que quantificou valores da ordem de $222,3 \mathrm{~kg} \mathrm{ha}^{-1}$ de K. A alta absorção e acúmulo de K nos colmos pode ser devido ao alto rendimento de colmos observado na canaplanta. Em relação ao efeito do sistema de colheita sobre a absorção e exportação de K, verificou-se que a cana colhida sem a queima da palhada apresentou exportação 27,6 \% superior à da cana queimada, indicando que a manutenção da palhada sobre o solo pode aumentar a disponibilidade e a eficiência de absorção desse nutriente pela cana-de-açúcar. 
Quadro 2. Nutrientes exportados nos colmos frescos e acumulados na matéria seca da palhada e parte aérea total de cana-planta colhida crua e queimada com diferentes doses de adubação

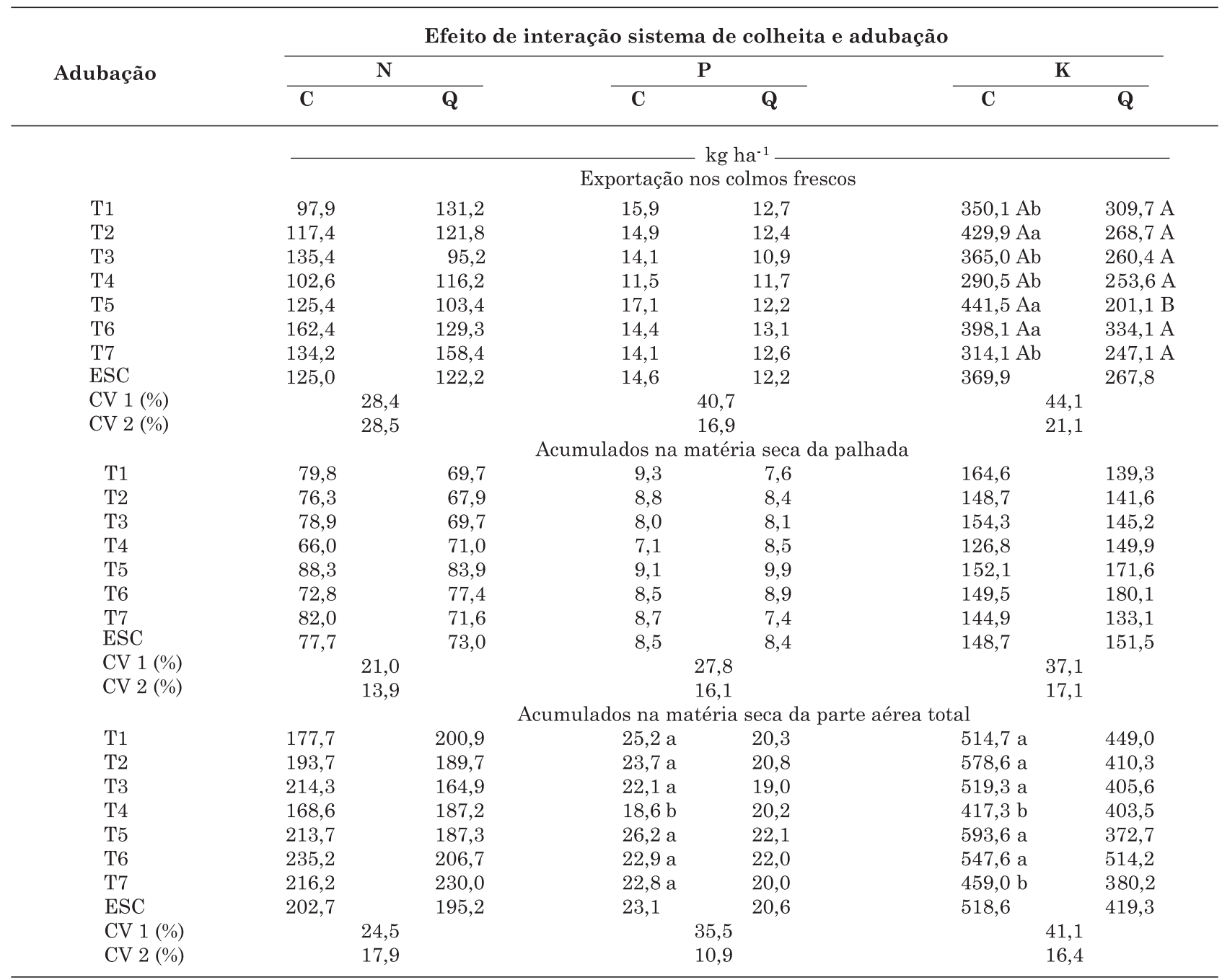

Valores médios de quatro repetições para efeito de interação e de 28 repetições para efeito de sistema de colheita. Letras maiúsculas diferem entre colunas e minúsculas entre linhas. A ausência de letras significa que não houve diferença entre as médias. T1: testemunha; T2: vinhaça; T3: vinhaça + $80 \mathrm{~kg}$ de N incorporado; T4: vinhaça + $80 \mathrm{~kg}$ de N em cobertura; T5: vinhaça + $40 \mathrm{~kg}$ de $\mathrm{N}$ em cobertura; T6: $\mathrm{K}_{2} \mathrm{O}+80 \mathrm{~kg}$ de $\mathrm{N}$ incorporado; T7: $\mathrm{K}_{2} \mathrm{O}+80 \mathrm{~kg}$ de $\mathrm{N}$ em cobertura. ESC: efeito de sistema de colheita. CV: coeficiente de variação. C: cana crua; Q: cana queimada. Teste de Scott-Knott a $5 \%$.

Em relação às quantidades de N, P e K acumulados na matéria seca da palhada, não foram verificadas diferenças entre os sistemas de colheita e os tratamentos com as diferentes adubações. Os valores de $\mathrm{N}$ acumulado na palhada foram superiores aos encontrados por Urquiaga et al. (1997) e Resende et al. (2006), que, estudando a ciclagem de nutrientes em cana-de-açúcar, observaram valores da ordem de $42 \mathrm{~kg} \mathrm{ha}^{-1}$ de N. Segundo Trivelin et al. (1996), os valores de nitrogênio da palhada podem oscilar de 40 a $80 \mathrm{~kg} \mathrm{ha}^{-1}$. Os valores de P e K acumulados na matéria seca da palhada corroboram os resultados observados por Orlando Filho (1993).

Avaliando o acúmulo total de N, P e K na parte aérea da cana-planta, verificou-se que não houve efeito de resíduos de adubações anteriores no acúmulo de
$\mathrm{N}$, ao passo que $\mathrm{P}$ e $\mathrm{K}$ foram responsivos à aplicação dos resíduos na cana crua. O acúmulo de $\mathrm{N}$ observado corrobora os resultados encontrados por Orlando Filho et al. (1980) e Urquiaga et al. (1992). Orlando Filho (1993), estudando o acúmulo de macronutrientes na cana-de-açúcar, constatou valores de $\mathrm{P}$ similares aos obtidos neste estudo. Este autor quantificou acúmulos de $\mathrm{K}$ da ordem de $174 \mathrm{~kg}$ de K para cada $100 \mathrm{t}^{-1}$ de colmos - valores relativamente menores que os verificados neste estudo. A cana cultivada no sistema de cana crua apresentou acúmulo de K na parte aérea $19,14 \%$ superior ao da cana cultivada com a queima da palhada. Esse resultado parece indicar que a manutenção da palhada sobre o solo após a colheita aumenta a disponibilidade e a absorção de $\mathrm{K}$ ao longo do ciclo vegetativo da cultura. Segundo Canellas et al. (2003), 
a adição de matéria orgânica na lavoura de cana-deaçúcar por longo prazo, por meio da preservação da palhada, proporciona melhoria na fertilidade do solo.

No quadro 3 são apresentados os rendimentos de colmos frescos e o acúmulo de palhada da cana-soca sob sistema de colheita crua e queimada, cultivada com diferentes doses de adubação. Avaliando o rendimento de colmos, somente do ponto de vista dos sistemas de colheita, observa-se que a cana crua foi superior à cana queimada. Segundo Mendoza et al. (2000), o aumento da produtividade da cana-de-açúcar no sistema de colheita de cana crua pode estar relacionado à manutenção da palhada sobre o solo, favorecendo a manutenção da sua umidade, aumentando o teor de matéria orgânica e a disponibilidade de nutrientes e, em decorrência disso, melhorando as propriedades físicas, químicas e biológicas do solo. Esses resultados reforçam a afirmação de Mielniczuk et al. (2003), de que a preservação da palhada sobre o solo aumenta a eficiência do sistema produtivo da cana-de-açúcar.

A análise do rendimento de colmos em função das adubações demonstra que a testemunha, o tratamento adubado somente com vinhaça e o tratamento com dose de $120 \mathrm{~kg}$ de $\mathrm{K}_{2} \mathrm{O}$ na forma de $\mathrm{KCl}+80 \mathrm{~kg} \mathrm{ha}^{-1}$ de $\mathrm{N}$ em cobertura foram inferiores às demais doses de adubação tanto na cana crua quanto na cana queimada. Os tratamentos que apresentaram os melhores rendimentos de colmos foram os que receberam a fertilização com vinhaça complementada

Quadro 3. Rendimento de colmos frescos e matéria seca de palhada de cana de primeira soca crua e queimada com diferentes doses de adubação

\begin{tabular}{|c|c|c|c|c|}
\hline \multirow{3}{*}{ Adubação } & \multicolumn{4}{|c|}{$\begin{array}{c}\text { Efeito de interação sistema } \\
\text { de colheita/adubação }\end{array}$} \\
\hline & \multicolumn{2}{|c|}{ Colmos } & \multicolumn{2}{|c|}{ Palhada } \\
\hline & Crua & Queimada & Crua & Queimada \\
\hline & \multicolumn{4}{|c|}{$\mathrm{tha}^{-1}$} \\
\hline $\mathrm{T} 1$ & $108,2 \mathrm{Ab}$ & $83,2 \mathrm{Bb}$ & $10,3 \mathrm{~b}$ & $11,9 \mathrm{a}$ \\
\hline $\mathrm{T} 2$ & $121,1 \mathrm{~b}$ & $100,0 \mathrm{~b}$ & $10,3 \mathrm{~b}$ & $11,8 \mathrm{a}$ \\
\hline T3 & $153,9 \mathrm{a}$ & $146,1 \mathrm{a}$ & $8,5 \mathrm{~b}$ & $13,0 \mathrm{a}$ \\
\hline $\mathrm{T} 4$ & $144,2 \mathrm{a}$ & $121,2 \mathrm{a}$ & $12,2 \mathrm{a}$ & $9,5 \mathrm{~b}$ \\
\hline $\mathrm{T} 5$ & $140,4 \mathrm{a}$ & $125,9 \mathrm{a}$ & $9,8 \mathrm{~b}$ & $13,1 \mathrm{a}$ \\
\hline $\mathrm{T} 6$ & $143,3 \mathrm{a}$ & $132,7 \mathrm{a}$ & $11,3 \mathrm{a}$ & $13,1 \mathrm{a}$ \\
\hline $\mathrm{T} 7$ & $121,2 \mathrm{~b}$ & $103,8 \mathrm{~b}$ & $9,5 \mathrm{~b}$ & $13,2 \mathrm{a}$ \\
\hline $\mathrm{ESC}$ & $133,2 \mathrm{~A}$ & $116,1 \mathrm{~B}$ & 10,3 & 12,2 \\
\hline CV $1(\%)$ & \multicolumn{2}{|c|}{11,2} & \multicolumn{2}{|r|}{39,2} \\
\hline CV $2(\%)$ & \multicolumn{2}{|c|}{14,8} & \multicolumn{2}{|r|}{10,4} \\
\hline
\end{tabular}

Valores médios de quatro repetições para efeito de interação $e$ de 28 repetições para efeito de sistema de colheita. Letras maiúsculas diferem entre colunas e minúsculas entre linhas. T1: testemunha; T2: vinhaça; T3: vinhaça $+80 \mathrm{~kg}$ de $\mathrm{N}$ incorporado; T4: vinhaça $+80 \mathrm{~kg}$ de $\mathrm{N}$ em cobertura; T5: vinhaça $+40 \mathrm{~kg}$ de $\mathrm{N}$ em cobertura; $\mathrm{T} 6: \mathrm{K}_{2} \mathrm{O}+80 \mathrm{~kg}$ de $\mathrm{N}$ incorporado; T7: $\mathrm{K}_{2} \mathrm{O}+80 \mathrm{~kg}$ de $\mathrm{N}$ em cobertura. ESC: efeito de sistema de colheita. CV: coeficiente de variação. Teste de Scott-Knott a $5 \%$. com $\mathrm{N}$ independentemente da forma de aplicação e o tratamento em que foi aplicada a dose de $120 \mathrm{~kg}$ de $\mathrm{K}_{2} \mathrm{O}$ na forma de $\mathrm{KCl}+80 \mathrm{~kg}$ de $\mathrm{N}$ incorporado ao solo. Em relação à complementação nitrogenada da vinhaça, verificou-se que a dose de $40 \mathrm{~kg}$ de $\mathrm{N}$ associada à vinhaça apresentou rendimento de colmos equivalente ao obtido com as doses de $80 \mathrm{~kg}$ de $\mathrm{N}$ associadas à vinhaça nos dois sistemas de colheita. Esse padrão pode ser justificado pela presença de $\mathrm{N}$ na vinhaça, que pode ter complementado a dose de $40 \mathrm{~kg}$ de N. Conforme Prada et al. (1998), a vinhaça proveniente do caldo, como é o caso deste estudo, pode conter de 150 a $700 \mathrm{mg} \mathrm{L}^{-1}$ de N. Com base nessa informação, a aplicação de $150 \mathrm{~m}^{3} \mathrm{ha}^{-1}$ de vinhaça pode adicionar de 22,5 a 105,0 kg ha-1 de $\mathrm{N}$. Os resultados indicam que o uso da vinhaça associada à adubação nitrogenada aplicada sobre o fertilizante incorporado ou não ao solo proporciona condições favoráveis para que a cana-de-açúcar expresse melhor seu potencial produtivo. Segundo Nunes et al. (1981), a vinhaça aumenta os teores de $\mathrm{Ca}^{2+}, \mathrm{Mg}^{2+} \mathrm{e} \mathrm{K}$ no solo, ao mesmo tempo em que a concentração de $\mathrm{Al}^{3+}$ decresce, resultando dessa forma em maior disponibilidade de nutrientes, aumento dos valores de $\mathrm{pH}$ do solo e, consequentemente, aumento da sua fertilidade. Almeida (1955) classificou a vinhaça como fator de fertilização ou de correção de solos, rico em matéria orgânica coloidal e em elementos minerais, que contribui para a elevação do $\mathrm{pH}$ dos solos, melhoria das suas propriedades químicas, físicas e biológicas e aumento da microflora dos solos, proporcionando mais fácil nitrificação, conferindo maior índice de fertilidade e propiciando à cana-de-açúcar condições mais favoráveis ao seu ciclo vegetativo. Rodrigues et al. (1984), baseando-se em resultados de grande número de ensaios de adubação nitrogenada em complementação

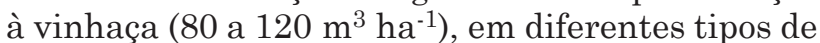
solo, recomendam doses de 90 a $100 \mathrm{~kg} \mathrm{ha}^{-1} \mathrm{de} \mathrm{N}$.

É importante destacar que os melhores resultados foram obtidos nos tratamentos em que a vinhaça foi associada à complementação nitrogenada e no tratamento que recebeu $120 \mathrm{~kg} \mathrm{ha}^{-1} \mathrm{de} \mathrm{K}_{2} \mathrm{O}$ na forma de $\mathrm{KCl}+80 \mathrm{~kg} \mathrm{ha}^{-1}$ de N incorporado ao solo (T6). Os resultados confirmam a importância da nutrição nitrogenada e potássica para a cana-de-açúcar e são corroborados por Camilotti et al. (2006) em estudos em que avaliaram a eficiência da vinhaça como fonte de K na produtividade da cana-de-açúcar.

$\mathrm{O}$ tratamento que recebeu $120 \mathrm{~kg} \mathrm{ha}^{-1} \mathrm{de} \mathrm{K}_{2} \mathrm{O}$ na forma de $\mathrm{KCl}+80 \mathrm{~kg} \mathrm{ha}^{-1}$ de $\mathrm{N}$ incorporado ao solo (T6) apresentou rendimento de colmos frescos da mesma magnitude que os tratamentos que receberam a vinhaça complementada com adubação nitrogenada, o que pode ser decorrente do melhor aproveitamento do fertilizante nitrogenado incorporado ao solo. Esse resultado é confirmado pela análise do rendimento de colmos do tratamento que recebeu $120 \mathrm{~kg} \mathrm{ha}^{-1} \mathrm{de} \mathrm{K}_{2} \mathrm{O}$ na forma de $\mathrm{KCl}+80 \mathrm{~kg} \mathrm{ha}^{-1}$ de $\mathrm{N}$ incorporado ao solo, sendo comparado ao tratamento que recebeu as mesmas doses de $\mathrm{N} \mathrm{e} \mathrm{K}$ (T7), porém neste tratamento 
o fertilizante foi aplicado em cobertura. Segundo Lara Gabezas (1998), estudando a volatilização de Nfertilizante (ureia) incorporado e em cobertura em sistema de plantio direto, a perda de $\mathrm{N}$ aplicado em cobertura pode atingir valores próximos a $70 \%$ do $\mathrm{N}$ total aplicado no solo, reduzindo dessa forma a eficiência da adubação e, consequentemente, o potencial produtivo da cultura de interesse.

A queda na produtividade de colmos da testemunha (T1) em relação aos demais tratamentos corrobora os resultados encontrados por Trivelin (2000) e Vitti (2003), para os quais a nutrição nitrogenada é fator determinante na produtividade e longevidade das soqueiras de cana-de-açúcar.
Em relação ao acúmulo de palhada, verificou-se que ocorreram diferenças significativas entre as adubações nos dois sistemas de colheita. O acúmulo de palhada observado assemelha-se aos resultados verificados por Vitti (2003), estudando a adubação nitrogenada da cana-de-açúcar soqueira colhida mecanicamente sem a queima prévia da palhada.

No quadro 4 são apresentados a exportação de N, $\mathrm{P}$ e K nos colmos frescos e o acúmulo de matéria seca de palhada e da parte aérea total de cana-soca.

A exportação de $\mathrm{N}$ nos colmos frescos não foi influenciada pelas adubações na cana crua; já para a queimada verificou-se influência das adubações nos valores de $\mathrm{N}$ exportados, porém as variações destes foram

Quadro 4. Nutrientes exportados nos colmos frescos e acumulados na matéria seca de palhada e parte aérea total de cana-soca colhida crua e queimada com diferentes doses de adubação

\begin{tabular}{|c|c|c|c|c|c|c|}
\hline \multirow{3}{*}{ Adubação } & \multicolumn{6}{|c|}{ Efeito de interação sistema de colheita e adubação } \\
\hline & \multicolumn{2}{|c|}{$\mathbf{N}$} & \multicolumn{2}{|c|}{$\mathbf{P}$} & \multicolumn{2}{|c|}{$\mathbf{K}$} \\
\hline & $\mathbf{C}$ & $\mathbf{Q}$ & $\mathbf{C}$ & $\mathbf{Q}$ & $\mathbf{C}$ & $\mathbf{Q}$ \\
\hline & \multicolumn{6}{|c|}{$-\mathrm{kg} \mathrm{ha}^{-1}$} \\
\hline & \multicolumn{6}{|c|}{ Exportação nos colmos frescos } \\
\hline $\mathrm{T} 1$ & $84,9 \mathrm{~A}$ & $59,8 \mathrm{Bb}$ & 12,4 & 11,6 & $285,5 \mathrm{~b}$ & $318,8 \mathrm{~b}$ \\
\hline $\mathrm{T} 2$ & 91,2 & $70,7 \mathrm{~b}$ & 19,2 & 11,4 & $420,1 \mathrm{a}$ & $345,5 \mathrm{~b}$ \\
\hline T3 & 94,4 & $99,0 \mathrm{a}$ & 15,1 & 18,9 & $420,5 \mathrm{a}$ & $535,5 \mathrm{a}$ \\
\hline $\mathrm{T} 4$ & 79,6 & $93,4 \mathrm{a}$ & 17,8 & 18,1 & $503,9 \mathrm{Aa}$ & $361,1 \mathrm{Bb}$ \\
\hline T5 & 108,7 & $97,8 \mathrm{a}$ & 25,1 & 18,3 & $468,2 \mathrm{a}$ & $472,6 \mathrm{a}$ \\
\hline $\mathrm{T} 6$ & 82,9 & $86,1 \mathrm{a}$ & 18,1 & 15,8 & $491,4 \mathrm{a}$ & $463,4 \mathrm{a}$ \\
\hline $\mathrm{T} 7$ & 63,9 & $79,4 \mathrm{~b}$ & 11,8 & 16,1 & $402,3 \mathrm{a}$ & $331,4 \mathrm{~b}$ \\
\hline $\mathrm{ESC}$ & 86,5 & 83,7 & 17,1 & 15,7 & 427,4 & 404,0 \\
\hline CV 1 (\%) & \multicolumn{2}{|c|}{18,4} & \multicolumn{2}{|c|}{38,7} & \multicolumn{2}{|c|}{17,6} \\
\hline \multirow[t]{2}{*}{ CV $2(\%)$} & \multicolumn{2}{|c|}{19,8} & \multicolumn{2}{|c|}{13,0} & \multicolumn{2}{|c|}{20,0} \\
\hline & \multicolumn{6}{|c|}{ Acumulados na matéria seca da palhada } \\
\hline $\mathrm{T} 1$ & 47,4 & 45,2 & 4,7 & 5,5 & 72,4 & $74,5 \mathrm{~b}$ \\
\hline $\mathrm{T} 2$ & 41,8 & 52,2 & 5,1 & 5,8 & 65,4 & $87,7 \mathrm{a}$ \\
\hline T3 & 35,6 & 50,3 & 4,8 & 5,7 & 66,5 & $77,2 \mathrm{~b}$ \\
\hline $\mathrm{T} 4$ & 47,5 & 40,7 & 5,1 & 4,4 & 70,2 & $59,7 \mathrm{c}$ \\
\hline T5 & 42,9 & 53,2 & 5,4 & 6,3 & 67,9 & 88,0 a \\
\hline T6 & 41,0 & 51,2 & 4,9 & 5,5 & 65,4 & $73,1 \mathrm{~b}$ \\
\hline $\mathrm{T} 7$ & 38,5 & 46,3 & 5,0 & 6,8 & 75,0 & $92,8 \mathrm{a}$ \\
\hline $\mathrm{ESC}$ & 42,1 & 48,4 & 5,0 & 5,7 & 69,0 & 79,0 \\
\hline CV $1(\%)$ & \multicolumn{2}{|c|}{42,9} & \multicolumn{2}{|c|}{30,3} & \multicolumn{2}{|c|}{23,5} \\
\hline \multirow[t]{2}{*}{ CV $2(\%)$} & \multicolumn{2}{|c|}{16,3} & \multicolumn{2}{|c|}{16,0} & \multicolumn{2}{|c|}{16,5} \\
\hline & \multicolumn{6}{|c|}{ Acumulados na matéria seca da parte aérea total } \\
\hline $\mathrm{T} 1$ & 132,3 & 105,0 & 17,1 & 17,1 & $357,9 \mathrm{~b}$ & $393,3 \mathrm{~b}$ \\
\hline $\mathrm{T} 2$ & 133,0 & 122,9 & 24,3 & 17,2 & $485,5 \mathrm{a}$ & $433,2 \mathrm{~b}$ \\
\hline T3 & 130,0 & 149,3 & 19,9 & 24,6 & $487,0 \mathrm{a}$ & $612,7 \mathrm{a}$ \\
\hline $\mathrm{T} 4$ & 127,1 & 134,1 & 22,9 & 22,5 & $574,1 \mathrm{Aa}$ & $420,8 \mathrm{Bb}$ \\
\hline T5 & 151,6 & 151,0 & 30,5 & 24,6 & $536,1 \mathrm{a}$ & $560,6 \mathrm{a}$ \\
\hline $\mathrm{T} 6$ & 123,9 & 137,3 & 23,0 & 21,3 & $556,8 \mathrm{a}$ & 536,5 a \\
\hline $\mathrm{T} 7$ & 102,4 & 125,7 & 16,8 & 22,9 & $477,3 \mathrm{a}$ & $424,2 \mathrm{~b}$ \\
\hline ESC & 128,6 & 132,2 & 22,1 & 21,4 & 496,4 & 483,0 \\
\hline CV $1(\%)$ & \multicolumn{2}{|c|}{23,9} & \multicolumn{2}{|c|}{27,6} & \multicolumn{2}{|c|}{17,5} \\
\hline CV $2(\%)$ & \multicolumn{2}{|c|}{16,1} & & & 17, & \\
\hline
\end{tabular}

Valores médios de quatro repetições para efeito de interação e de 28 repetições para efeito de sistema de colheita. Letras maiúsculas diferem entre colunas e minúsculas entre linhas. A ausência de letras significa que não houve diferença entre as médias. T1: testemunha; T2: vinhaça; T3: vinhaça $+80 \mathrm{~kg}$ de $\mathrm{N}$ incorporado; T4: vinhaça $+80 \mathrm{~kg}$ de $\mathrm{N}$ em cobertura; T5: vinhaça + $40 \mathrm{~kg}$ de $\mathrm{N}$ em cobertura; T6: $\mathrm{K}_{2} \mathrm{O}+80 \mathrm{~kg}$ de $\mathrm{N}$ incorporado; $\mathrm{T} 7: \mathrm{K}_{2} \mathrm{O}+80 \mathrm{~kg}$ de $\mathrm{N}$ em cobertura. ESC: efeito de sistema de colheita. CV: coeficiente de variação. C: cana crua; Q: cana queimada. Teste de Scott-Knott a $5 \%$. 
determinadas pelos rendimentos de colmos. A exportação de $\mathrm{N}$ diferiu entre os sistemas de colheita para a testemunha. A cana crua proporcionou maior acúmulo de $\mathrm{N}$ nos colmos. A exportação de $\mathrm{P}$ nos colmos não foi alterada pelos sistemas de colheita $\mathrm{e}$ pelas diferentes doses de adubação. Diferentemente do observado para o $\mathrm{P}$, a exportação de $\mathrm{K}$ foi influenciada pelos sistemas de colheita e pelas doses de adubação. Ainda quanto ao K, destaca-se que os seus menores valores de exportação ocorreram na testemunha. Os resultados na exportação de K evidenciam que a absorção deste nutriente é fator determinante na produção de cana-de-açúcar. Segundo Ernani et al. (2007), o K tem inúmeras funções na planta, destacando-se, principalmente, a ativação de vários sistemas enzimáticos, muitos deles participantes dos processos de fotossíntese e respiração. A exportação de $\mathrm{N}$ e P observada neste estudo corrobora os valores encontrados por Malavolta et al. (1997), avaliando o estado nutricional da cana-de-açúcar; quanto aos valores de exportação de K, estes foram superiores aos observados por Oliveira (2008). Este autor, estudando a dinâmica de nutrientes em cana-de-açúcar em um sistema irrigado de produção utilizando a mesma variedade deste estudo, verificou a exportação de 222,3 $\mathrm{kg} \mathrm{ha}^{-1}$ de K.

Quanto ao acúmulo de N, P e K na palhada da canasoca, somente o $\mathrm{K}$ foi influenciado pelas doses de adubação na cana queimada. Os acúmulos observados são corroborados por vários autores, que avaliaram a nutrição e o acúmulo de nutrientes em cana-de-açúcar (Orlando Filho, 1993; Trivelin et al., 1996; Oliveira et al., 1999).

No acúmulo de N, P e K na parte aérea total da cana-de-açúcar, verificou-se que o $\mathrm{K}$ apresentou oscilações entre as adubações nos dois sistemas de colheita. É importante destacar que a testemunha (T1) apresentou os menores acúmulos de K na parte aérea total, nos dois sistemas de colheita. Esse resultado leva a inferir que a não reposição deste nutriente no solo contribui para redução da longevidade e produtividade do canavial. Em todos os tratamentos, os valores de $\mathrm{K}$ acumulado foram altos, quando comparados aos obtidos por vários autores citados anteriormente. Oliveira (2008), estudando a dinâmica de nutrientes em cana-de-açúcar em sistema irrigado de produção com a mesma variedade (RB 867515), verificou acúmulo total de K (parte aérea e subterrânea) semelhante ao observado somente na parte aérea neste estudo. Esse padrão indica que o acúmulo de elevada quantidade de potássio parece ser uma característica intrínseca da variedade.

\section{CONCLUSÕES}

1. Não foi verificado efeito residual das adubações do ciclo anterior no rendimento de colmos na canaplanta.
2. A adubação da cana-de-açúcar com vinhaça associada à complementação nitrogenada e a adubação com $\mathrm{K}_{2} \mathrm{O}$ do $\mathrm{KCl}$ associada à complementação nitrogenada incorporada ao solo proporcionaram os melhores rendimentos de colmos frescos.

3. As doses de 40 e $80 \mathrm{~kg} \mathrm{ha}^{-1}$ de $\mathrm{N}$ associadas à fertilização com vinhaça apresentaram rendimentos equivalentes de colmos.

4. O sistema de colheita cana crua proporcionou rendimento de colmos superior ao da cana queimada na primeira soca.

\section{LITERATURA CITADA}

ALMEIDA, J.R. O problema da vinhaça. Brasil Açuc., 46:72$77,1955$.

AMARAL SOBRINHO, N.M.B. Efeito da vinhaça em mistura com nitrato na dinâmica do nitrogênio em solo ácido. Itaguaí, Universidade Federal Rural do Rio de Janeiro, 1983. 95p. (Tese de Doutorado)

AZEREDO, D.F.; BOLSANELLO, J.; WEBER, H. \& VIEIRA, J.R. Nitrogênio em cana-planta - doses e fracionamento. R. STAB, 6:26-33, 1986.

BODDEY, R.M.; POLIDORO, J.C.; RESENDE, A.S.; ALVES, B.J.R. \& URQUIAGA, S. Use of the ${ }^{15} \mathrm{~N}$ natural abundance technique for the quantification of the contribution of $\mathrm{N}_{2}$ fixation to sugar cane and other grasses. Austr. J. Plant Physiol., 28:1-7, 2001.

CAMILOTTI, F.; ANDRIOLI, I.; MARQUES, M.O.; SILVA, A.R.; TASSO JUNIOR, L.C.; NOBILE, F.O.; NOGUEIRA, G.A. \& PRATI, F. Produtividade e qualidade agroindustrial da cana-de-açúcar cultivada com lodo de esgoto, vinhaça e adubos minerais. R. STAB: Açúcar, Álcool Sub. 24:32-35, 2006.

CANELLAS, L.P.; VELLOSO, A.C.X.; MARCIANO, C.R.; RAMALHO, J.F.G.P.; RUMJANEK, V.M.; REZENDE, C.E. \& SANTOS, G.A. Propriedades químicas de um Cambissolo cultivado com cana-de-açúcar, com preservação do palhiço e adição de vinhaça por longo tempo. R. Bras. Ci. Solo, 27:935-944, 2003.

DE-POLLI, H.; ALMEIDA, D.L.; SANTOS, G.A.; CUNHA, L.H.; FREIRE, L.R.; AMARAL SOBRINHO, N.M.B.; PEREIRA, N.N.C.; EIRA, P.A.; BLOISE, R.M. \& SALER, R.C. Manual de adubação para o Estado do Rio de Janeiro. Itaguaí, Universidade Federal Rural do Rio de Janeiro, 1988. 179p. (Série Ciências Agrárias, 2)

DÖBEREINER, J.; DAY, J.M. \& DART, P.J. Nitrogenase activity in the rhizosphere of sugar cane and some other tropical grasses. Plant Soil, 37:191-196, 1972.

ERNANI, P.R.; ALMEIDA, J.A. \& SANTOS, F.C. Potássio. In: NOVAIS, R. F.; ALVAREZ V., V.H.; BARROS, N.F.; FONTES, R.L.F.; CANTARUTTI, R.B. \& NEVES, J.C.L. Fertilidade do solo. Viçosa, MG, Sociedade Brasileira de Ciência do Solo, 2007. p.551-594. 
FREIRE, W.J. \& CORTEZ, 1.A.B. Vinhaça de cana-de-açúcar. Guaíba, Agropecuária, 2000. 203p. (Série Engenharia Agrícola, 1)

GLÓRIA, N. \& ORLANDO FILHO, J. Aplicação de vinhaça: Um resumo e discussões sobre o que foi pesquisado. $R$. Álcool Açúcar, 16:32-39, 1984.

INSTITUTO BRASILEIRO DE GEOGRAFIA E ESTATÍSTICA - IBGE. Levantamento Sistemático da Produção Agrícola (2006), Sistema IBGE e recuperação automática - SIDRA. Disponível em:< http:// www.ibge.gov.br > Acesso em: 25 de jan. de 2010.

LARA CABEZAS, W.A.R. Comportamento dos adubos nitrogenados em clima e solo de Cerrado. In: CURSO SOBRE ASPECTOS BÁSICOS DE FERTILIDADE E MICROBIOLOGIA DO SOLO SOB PLANTIO DIRETO, 1., Passo Fundo, 1998. Curso... Passo Fundo, Aldeia Norte, 1998. p.78-92.

LEAL, J.R.; AMARAL SOBRINHO, N.M.B.; VELLOSO, A.C.X. \& ROSSIELLO, R.O.P. Potencial redox e pH: Variações em um solo tratado com vinhaça. R. Bras. Ci. Solo, 7:257$261,1983$.

LIMA, J.F.W.F.; DA SILVA, G.L.; DE LUNA, J.G.; DA SILVA, M.A. \& OLIVEIRA, E.M. Efeito de doses crescentes de vinhaça e níveis de nitrogênio na produtividade da canade-açúcar. In: CONGRESSO NACIONAL DA STAB, 4., Olinda, 1987. Anais... Olinda, 1987. p.730-737.

MALAVOLTA, E.; VITTI, G.C. \& OLIVEIRA, S.A. Avaliação do estado nutricional das plantas. Piracicaba, Associação Brasileira para a Pesquisa da Potassa e do Fosfato, 1989. 201p.

MALAVOLTA, E.; VITTI, G.C. \& OLIVEIRA, S.A. Avaliação do estado nutricional das plantas: Princípios e aplicações. Piracicaba, Potafós, 1997. 319p.

MENDOZA, H.N.S.; LIMA, E.; SILVA, L.A. \& ANJOS, L.H.C. Propriedades químicas e biológicas de solo de tabuleiro cultivado com cana-de-açúcar com e sem queima da palhada. R. Bras. Ci. Solo, 24:201-207, 2000.

MIELNICZUK, J.; BAYER, C.; VEZZANI, F.M.; LOVATO, T.; FERNANDES, F.F. \& DEBARBA, L. Manejo de solo e culturas e sua relação com os estoques de carbono e nitrogênio do solo. In: NOVAIS, R.F.; ALVAREZ V., V.H. \& SCHAEFER, C.E.G.R., eds. Tópicos em ciência do solo. Viçosa, MG, Sociedade Brasileira de Ciência do Solo, 2003. p.209-248.

NUNES, M.R.; VELLOSO, A.C.X. \& LEAL, J.R. Efeito da vinhaça nos cátions trocáveis e outros elementos químicos do solo. Pesq. Agropec. Bras., 16:171-176, 1981.

OLIVEIRA, M.W.; TRIVELIN, P.C.O.; PENATTI, C.P. \& PICCOLO, M.C. Decomposição e liberação de nutrientes da palhada de cana-de-açúcar em campo. Pesq. Agropec. Bras., 34, p.2359-2362, 1999. (Notas científicas)

OLIVEIRA, M.W.; MENDE, L.C.; BARBOSA, M.H.P.; VITTI, A.C. \& FARIA, R.O. Avaliação do potencial produtivo de sete variedades de cana-de-açúcar sob irrigação complementar. Inf. Agron., 101:9-10, 2003.
OLIVEIRA, E.C.A. Dinâmica de nutrientes em cana-de-açúcar em sistema irrigado de produção. Recife, Universidade Federal Rural de Pernambuco, 2008. 76p. (Tese de Mestrado)

ORLANDO FILHO, J.; ZAMBELLO JUNIOR, E. \& AGUJARU, R. Efeito da aplicação prolongada da vinhaça nas propriedades químicas dos solos com cana-de-açúcar: Estudo exploratório. R. STAB, 1:28-33, 1983.

ORLANDO FILHO, J.; HAAG, H.P. \& ZAMBELLO Jr., E. Crescimento e absorção de macronutrientes pela canade-açúcar, variedade CB 41-76 em função de idade em solos do Estado de São Paulo. Piracicaba, Planalsucar, 1980. 128p. (Boletim Técnico, 2)

ORLANDO FILHO, J. Calagem e adubação da cana-de-açúcar. In: CÂMARA, G.M.S. \& OLIVEIRA, E.A.M. Produção de cana-de-açúcar. 9.ed. Piracicaba, FEALQ/USP, 1993. p.133146.

PROGRAMA DE MELHORAMENTO GENÉTICO DA CANADE-AÇÚCAR - UNIVERSIDADE FEDERAL DE VIÇOSA - PMGCA-UFV. Disponível em:< http:// pmgca.dbv.cca.ufscar.br > Acesso em: 27 fev. 2008.

PRADA, S.M. et al. Metodologia analítica para a determinação de sulfato em vinhoto. Química Nova, 21:249-252, 1998.

RESENDE, A.S.; SANTOS, A.; XAVIER, R.P.; COELHO, C.H.; GONDIM, A.; OLIVEIRA, O.C.; ALVES, B.J.R.; BODDEY, R.M. \& URQUIAGA, S. Efeito da queima da palhada da cana-de-açúcar e de aplicações de vinhaça e adubo nitrogenado em características tecnológicas da cultura. R. Bras. Ci. Solo, 30:937-941, 2006.

RODRIGURES, J.C.S.; PENNA, M.J. \& MORAES, R.S. Complementação nitrogenada em áreas fertilizadas com vinhaça. In: REUNIÃO TÉCNICA AGRONÔMICA: MANEJO E ADUBAÇÃO NA CULTURA DA CANA-DEAÇÚCAR, 3., Piracicaba, 1984. Anais... Piracicaba, COPERSUCAR, 1984. p.180-195.

SEGATO, S.V.; PINTO, A.S.; JENDIROBA, E. \& NÓBREGA, J.C.M. Atualização em produção de cana-de-açúcar. Piracicaba, Gráfica Prol, 2006. 415p.

TRIVELIN, P.C.O.; RODRIGUÊS, J.C.S. \& VICTORIA, R.L. Utilização por soqueira de cana-de açúcar de início de safra do nitrogênio da aquamônia-15N e ureia-15N aplicado ao solo em complemento à vinhaça. Pesq. Agropec. Bras., 31:89-99, 1996.

TRIVELIN, P.C.O. Utilização do nitrogênio pela cana-deaçúcar: Três casos estudados com o uso do traçador ${ }^{15} \mathrm{~N}$. Piracicaba, Universidade de São Paulo, 2000. 143p. (Tese de Livre-Docência)

URQUIAGA, S.; CRUZ, K.H.S. \& BODDEY, R.M. Contribution of nitrogen fixation to sugar cane: Nitrogen-15 and nitrogen balance estimates. Soil Sci. Soc. Am. J., 56:105114,1992

URQUIAGA, S.; RESENDE, A.S.; QUESADA, D.M.; SALLES, L.; GONDIM, A.; ALVES, B.J.R., \& BODDEY, R.M. Efeito das aplicações de vinhaça, adubo nitrogenado e da queima no rendimento de cana-de-açúcar. In: CONGRESSO BRASILEIRO DE CIÊNCIA DO SOLO, 26., Rio de Janeiro, 1997. Anais. Rio de Janeiro, Sociedade Brasileira de Ciência do Solo, 1997. CD-ROM. 
URQUIAGA, S.; LIMA, R.M.; XAVIER, R.P.; RESENDE, A.S.; ALVES, B.J.R. \& BODDEY, R.M. Avaliação da eficiência do processo de fixação biológica de nitrogênio em diferentes variedades de cana-de-açúcar. Agronomia, $37: 55-58,2003$
VITTI, A.C. Adubação nitrogenada da cana-de-açúcar (soqueira) colhida mecanicamente sem a queima prévia: Manejo e efeito na produtividade. Piracicaba, Universidade de São Paulo, Centro de Energia Nuclear na Agricultura, 2003. 114p. (Tese de Doutorado) 